\section{Conocimiento de métodos anticonceptivos en estudiantes adolescentes}

\author{
Knowledge of contraceptive methods in adolescent students \\ Conhecimento de métodos contraceptivos em estudantes
adolescentes
}

\author{
Ximena Lorena Carpio \\ loreaxs@gmail.com
}

Recibido septiembre 2019 / Revisión octubre 2019 / Aceptado 1 de enero 2020

\section{RESUMEN}

Introducción: La adolescencia según la Organización Mundial de la Salud es el periodo de crecimiento y desarrollo que se produce después de la niñez y antes de la edad adulta, comprende desde los 12 a 19 años de edad. Objetivo: Determinar el nivel de conocimiento acerca de los métodos anticonceptivos que tienen los adolescentes de las Unidades Educativas "Cañaviri y Santiago de Ventilla". Material y métodos: Se realizó un estudio cuantitativo, descriptiva, observacional y de corte transversal, con una población y muestra constituida por 81 adolescentes de las unidades educativas. Resultados: En relación a los conocimientos demostrados los de clase alta representaba poco porcentaje de la población y contestaron de manera correcta, el gran porcentaje estaba compuesto por adolescente de medianos y bajos recursos, los de recursos medio contestaron en un $41,9 \%$ de forma incorrecta mientras que los de bajo recursos contestaron de forma incorrecta en un 17,20\%. En cuanto al conocimiento de métodos anticonceptivos por sexo, la población de sexo femenino demostró según los porcentajes poseer más conocimiento acerca del tema. Conclusiones: El nivel de conocimiento de los métodos anticonceptivos es limitado, lo que demuestra que los adolescentes están expuestos a enfermedades de transmisión sexual y a embarazos prematuros.

Palabras clave: Métodos anticonceptivos; adolescencia; sexualidad

\section{ABSTRACT}

Introduction: Adolescence according to the World Health Organization is the period of growth and development that occurs after childhood and before adulthood, it ranges from 12 to 19 years of age. Objective: To determine the level of knowledge about the contraceptive methods that adolescents of the Educational Units "Cañaviri and Santiago de Ventilla" have. Material and methods: A quantitative, descriptive, observational and cross-sectional study was carried out with a population and sample made up of 81 adolescents from the educational units. Results: In relation to the knowledge shown, the upper class represented a small percentage of the population and answered correctly, the large percentage was made up of adolescents of medium and low resources, those of medium resources answered in a $41.9 \%$ way. incorrect, while those with low resources answered incorrectly by $17.20 \%$. Regarding knowledge of contraceptive methods by sex, the female population showed, according to the percentages, to have more knowledge about the subject. Conclusions: The level of knowledge of contraceptive methods is limited, which shows that adolescents are exposed to sexually transmitted diseases and premature pregnancies.

Key words: contraceptive methods; adolescence; sexuality
XC: Universidad Mayor de San Andrés. Bolivia. 


\section{RESUMO}

XC: Universidad Mayor de San Andrés. Bolivia.
Introdução: A adolescência, segundo a Organização Mundial da Saúde, é o período de crescimento e desenvolvimento que ocorre após a infância e antes da idade adulta, varia de 12 a 19 anos. Objetivo: Determinar o nível de conhecimento sobre os métodos contraceptivos que os adolescentes das Unidades Educacionais "Cañaviri e Santiago de Ventilla" possuem. Material e métodos: Foi realizado um estudo quantitativo, descritivo, observacional e transversal, com população e amostra composta por 81 adolescentes das unidades educacionais. Resultados: em relação ao conhecimento apresentado, a classe alta representou uma pequena porcentagem da população e respondeu corretamente, a grande porcentagem foi composta por adolescentes de recursos médios e baixos, os de recursos médios responderam de $41,9 \%$. incorretos, enquanto aqueles com poucos recursos responderam incorretamente em $17,20 \%$. Quanto ao conhecimento dos métodos contraceptivos por sexo, a população feminina mostrou, de acordo com os percentuais, maior conhecimento sobre o assunto. Conclusões: O nível de conhecimento dos métodos contraceptivos é limitado, o que mostra que os adolescentes são expostos a doenças sexualmente transmissíveis e gestações prematuras.

Palavras-chave: Métodos contraceptivos; adolescencia; sexualidade

\section{INTRODUCCIÓN}

$\mathrm{E}$ l control de la natalidad es una preocupación en todas las épocas de la historia de la humanidad, por lo que se realiza numerosos esfuerzos orientados a la búsqueda de métodos que ayuden a la mujer a pensar y vivir la maternidad, no como su destino, sino como una opción. Cada minuto muere en el mundo una mujer como consecuencia de un aborto o un parto atendido en condiciones desfavorables y cientos de las que no fallecen quedan mutiladas debido a complicaciones de estos procedimientos (1).

La adolescencia según la Organización Mundial de la Salud (OMS) es el periodo de crecimiento y desarrollo que se produce después de la niñez y antes de la edad adulta, comprende desde los 15 a 19 años de edad, es en este periodo donde se experimenta cambios físicos, psicológicos y sociales, así también desarrollan habilidades y conocimientos que ayudaran a la toma de decisiones en la vida adulta; además de que producen cambios propios de la sexualidad y la reproducción que constantemente se encuentra influenciada por el entorno biológico, ambiental, psicológico y social (2).

El embarazo no deseado y el riesgo de adquirir una Infección de Transmisión Sexual (ITS), el aborto inducido, morbilidad, discapacidad y mortalidad materna e infantil, son unas de las principales consecuencias del inicio de la actividad sexual sin protección y a edades precoces. La fecundidad temprana acarrea consigo todo tipo de consecuencia para los jóvenes a quienes dificulta continuar con sus estudios y conseguir un empleo.

A nivel global, una gran cantidad de adolescentes son sexualmente activos y el $60 \%$ no utiliza ningún método anticonceptivo (MAC). Cada año dan a luz 16 millones de adolescentes en el mundo, el $90 \%$ de estos embarazos ocurre en países en desarrollo, de estos 38\% sucede en América Latina y el Caribe (3).

Las relaciones sexuales en la adolescencia se caracterizan por ser irregulares, espaciadas y, con frecuencia, no previstas. La dificultad de acceso a los servicios sanitarios, la menor percepción del riesgo y la deficiente educación sexual, entre otros factores, sitúan a las 
adolescentes en una situación de alto riesgo de embarazos no planificados y de infecciones de transmisión sexual (ITS). La elección de un método contraceptivo requiere de un cuidadoso asesoramiento médico. Es necesario que los adolescentes conozcan todos los métodos de anticoncepción reversibles para poder elegir el que mejor se adapte a sus necesidades, valores, preferencias $\mathrm{y}$ personalidad $(4,5)$.

En la última revisión la OMS ha establecido 1870 recomendaciones en total, con 86 recomendaciones nuevas y 165 recomendaciones revisadas, lo que implica un esfuerzo en actualizar al máximo los criterios de elegibilidad, quedan establecidas en cuatro categorías según el riesgo que conlleva utilizar cada método en cada situación, también teniendo en cuenta si es el inicio del tratamiento o la continuación del mismo $(6,7)$.

Los métodos anticonceptivos en la adolescencia se pueden clasificar en: recomendables/preferentes: preservativo, anticoncepción hormonal combinada (oral, transdérmica y anillo vaginal), anticoncepción hormonal oral de solo gestágeno y dispositivo intrauterino (DIU). Aceptables: anticoncepción hormonal de depósito (inyectables e implantes) o diafragma. Poco aceptables: métodos naturales y contracepción quirúrgica. De emergencia: contracepción poscoital.

En la reciente guía sobre prevención del embarazo en la adolescente, la Academia Americana de Pediatría recomienda los anticonceptivos reversibles de acción prolongada: dispositivos intrauterinos, implantes subdérmico y acetato de medroxiprogesterona ofrecen una protección anticonceptiva de varios años y son seguros para la adolescente (5).

Las contraindicaciones de anticoncepción en adolescentes prácticamente no existen, considerando que esta población es habitualmente sana. En general, las contraindicaciones según enfermedades son las descritas en los criterios de elegibilidad de la Organización Mundial de la Salud. La principal recomendación es que cada caso debe ser observado en forma individual.

Dentro de las situaciones clínicas que pueden darse en la adolescencia y que contraindican el uso de métodos anticonceptivos solo hormonales se encuentran: trombofilia hereditaria, enfermedad tromboembólica venosa, síndrome de anticuerpo antifosfolipidicos, cirugía mayor e inmovilización prolongada (8).

\section{MATERIALES Y MÉTODO}

S e realizó un estudio cuantitativo con modalidad descriptiva, observacional y de corte transversal, con una población y muestra constituida por 81 adolescentes de las unidades educativas "Cañaviri y Santiago de Ventilla", con edades comprendidas entre 14 y 19 años de edad. La recolección de datos se realizó por medio de un cuestionario aplicado a los adolescentes de forma anónima, con previo consentimiento informado de los padres $y$ todo el tratamiento bioético pertinente.

\section{RESULTADOS Y DISCUSIÓN}

$\mathrm{U}$

n punto importante a considerar en el tema relacionado con el conocimiento por parte de los adolescentes de los métodos anticonceptivos es la convivencia de los adolescentes ya que de esto depende mucho su educación, valores inculcados. 
Tabla 1. Frecuencia y porcentaje según edad

\begin{tabular}{llll}
\hline Variable & Rango & Frecuencia & Porcentaje \\
\hline \multirow{2}{*}{ Edad } & $14-16$ & 44 & $55 \%$ \\
& $17-19$ & 37 & $45 \%$ \\
\hline
\end{tabular}

Fuente: Elaboración Propia

Tabla 2. Porcentaje y frecuencia según sexo de la población estudiada

\begin{tabular}{llll}
\hline Variable & Rango & Frecuencia & Porcentaje \\
\hline Con quien vives & Ambos padres & 68 & $84 \%$ \\
& Madre & 8 & $10 \%$ \\
& Padre & 5 & $6 \%$ \\
& Otros & 2 & $3 \%$ \\
\hline
\end{tabular}

Fuente: Elaboración propia

Tabla 3. Convivencia del adolescente

\begin{tabular}{llll}
\hline Variable & Rango & Frecuencia & Porcentaje \\
\hline Sexo & Femenino & 45 & $56 \%$ \\
& Masculino & 36 & $44 \%$ \\
\hline
\end{tabular}

Fuente: Elaboración propia

Se realizó la distribución de la población según nivel socioeconómico en alto, medio y bajo considerando en este último grupo a aquellas personas con déficit para adquisición de alimentos, acceso a servicios públicos y cubrir otras necesidades básicas. 


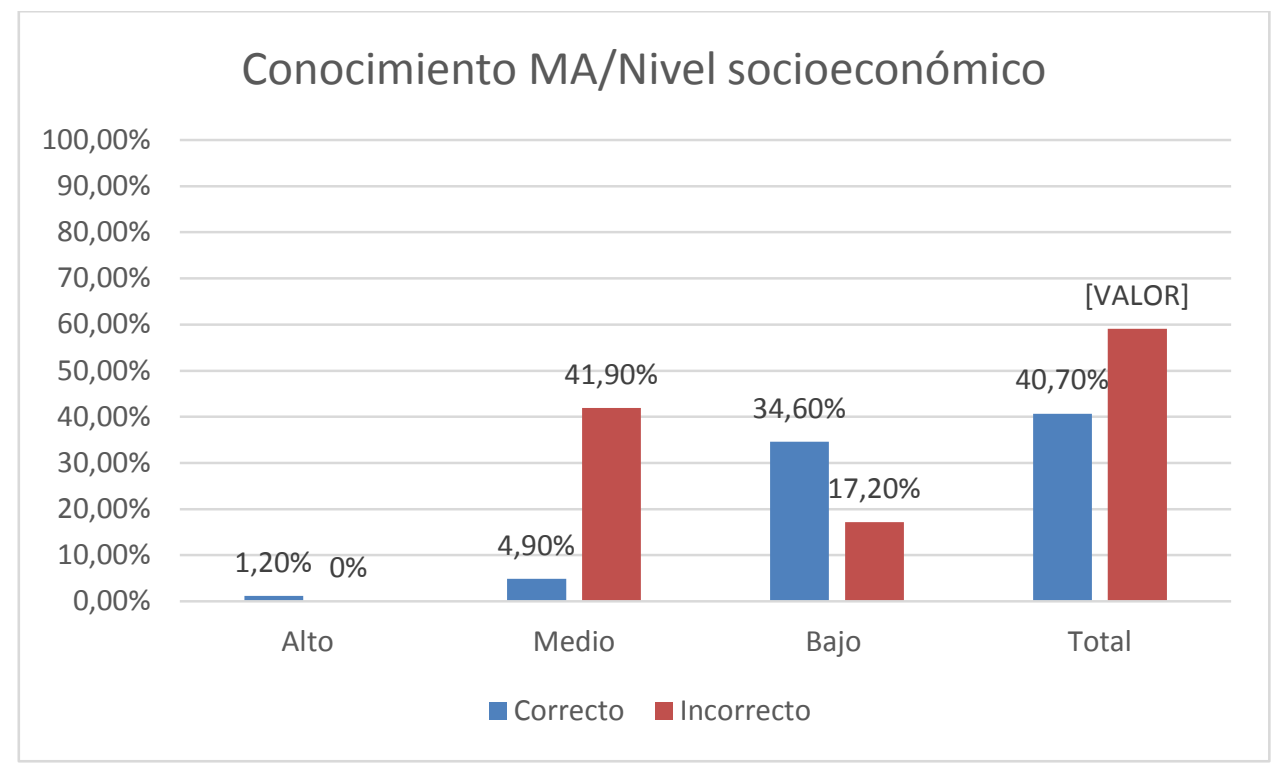

Gráfico 1. Nivel de conocimiento de los adolescentes relacionado con el nivel socioeconómico.

$\mathrm{Al}$ establecer la relación del conocimiento con el nivel socioeconómico se obtuvo que los adolescentes de nivel alto eran muy pocos y los que se consideraron dentro del estudio poseían conocimientos acerca de los métodos anticonceptivos (MAC), en el grupo considerado con un nivel socioeconómico medio representaron el $46,8 \%$ del total de la población encuestada, de estos solo
4,9\% manejaba la información correcta con respecto a los MAC y el restante $41,9 \%$ manejaba la información de manera incorrecta. En cuanto a la población de bajos recursos constituyó un $51,8 \%$ de la población objeto de esta investigación de los cuales el 34,6\% tenía información correcta acerca de los métodos anticonceptivos y el restante $17,2 \%$ poseía información incorrecta de estos.

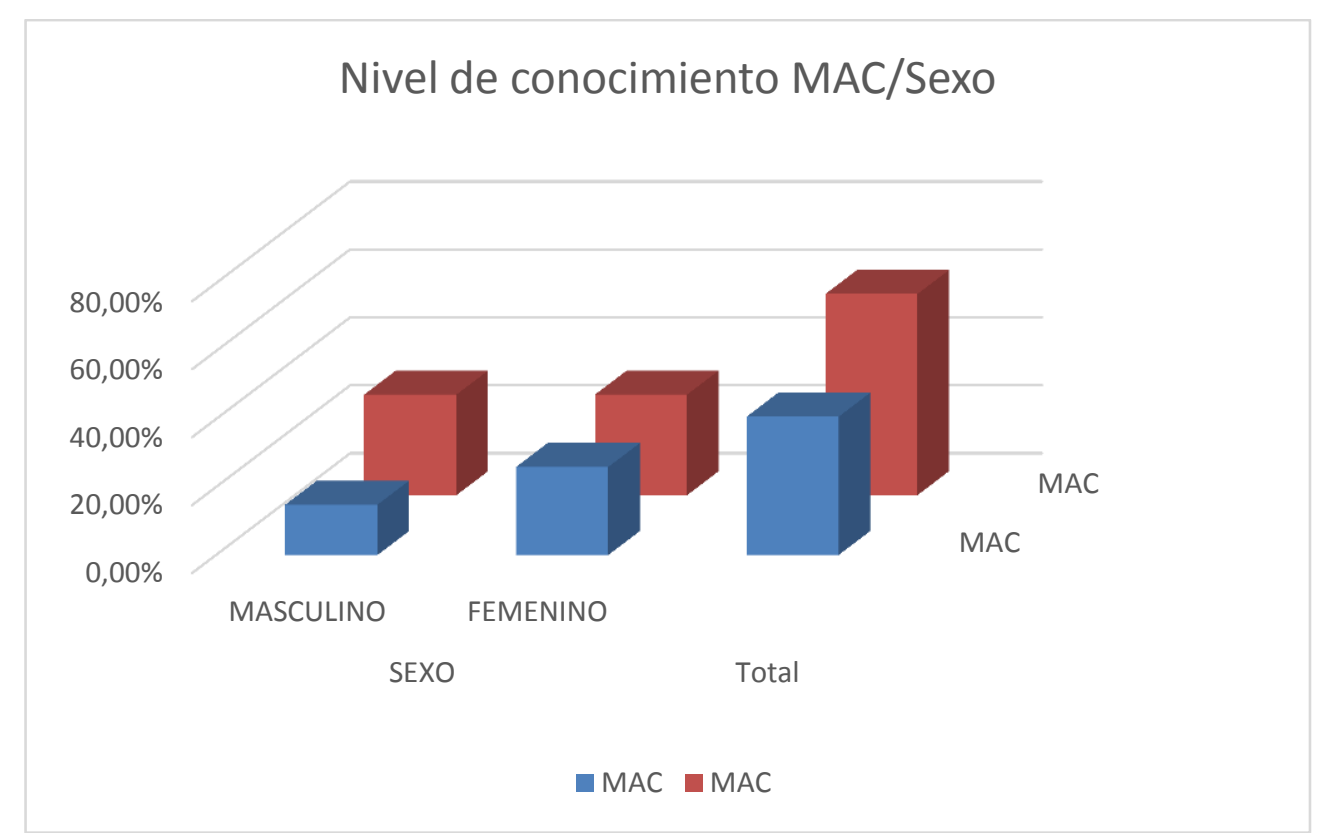

Gráfico 2. Nivel de conocimiento de los métodos anticonceptivos relacionado con el sexo. 
Se estableció la relación acerca del conocimiento de los MAC con el sexo y se obtuvo como resultado que de un total de 81 adolescentes quienes representan el 100\% de la población estudiada representando el sexo femenino un total de $55 \%$ de los cuales con respecto al conocimiento de los métodos anticonceptivos poseían los conocimientos de manera correcta en un 26\% mientras que el restante 19\% tenía la información de forma incorrecta. En el sexo masculino se obtuvo que un 14,8\% poseía la información de manera adecuada y el restante tenía la información de forma incorrecta.

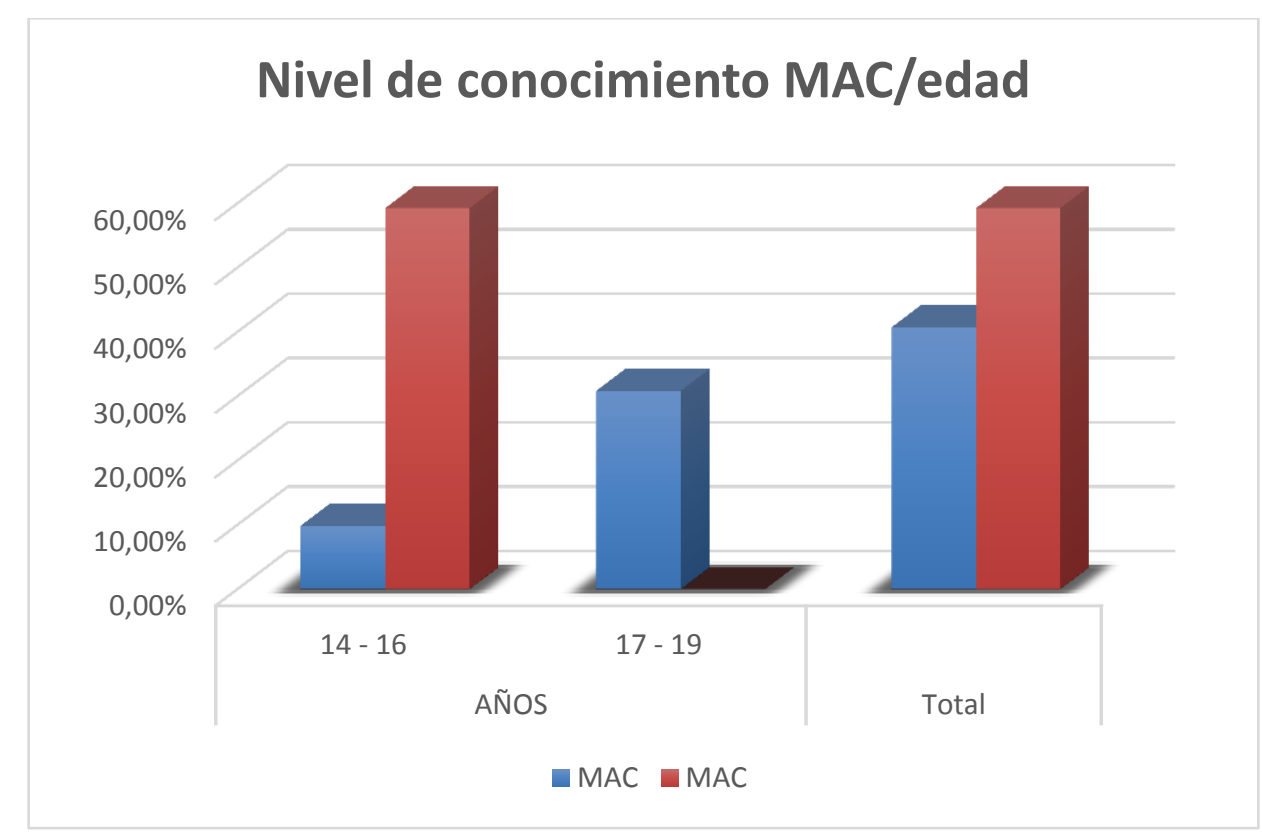

Gráfico 3. Nivel de conocimiento de los métodos anticonceptivos relacionado con la edad.

La población adolescente en este estudio se dividió en dos: los adolescentes de 14 a 16 años de edad y de 17 a 19 años, se encontró que en ambos grupos la mayoría maneja la información acerca de los MAC de manera incorrecta

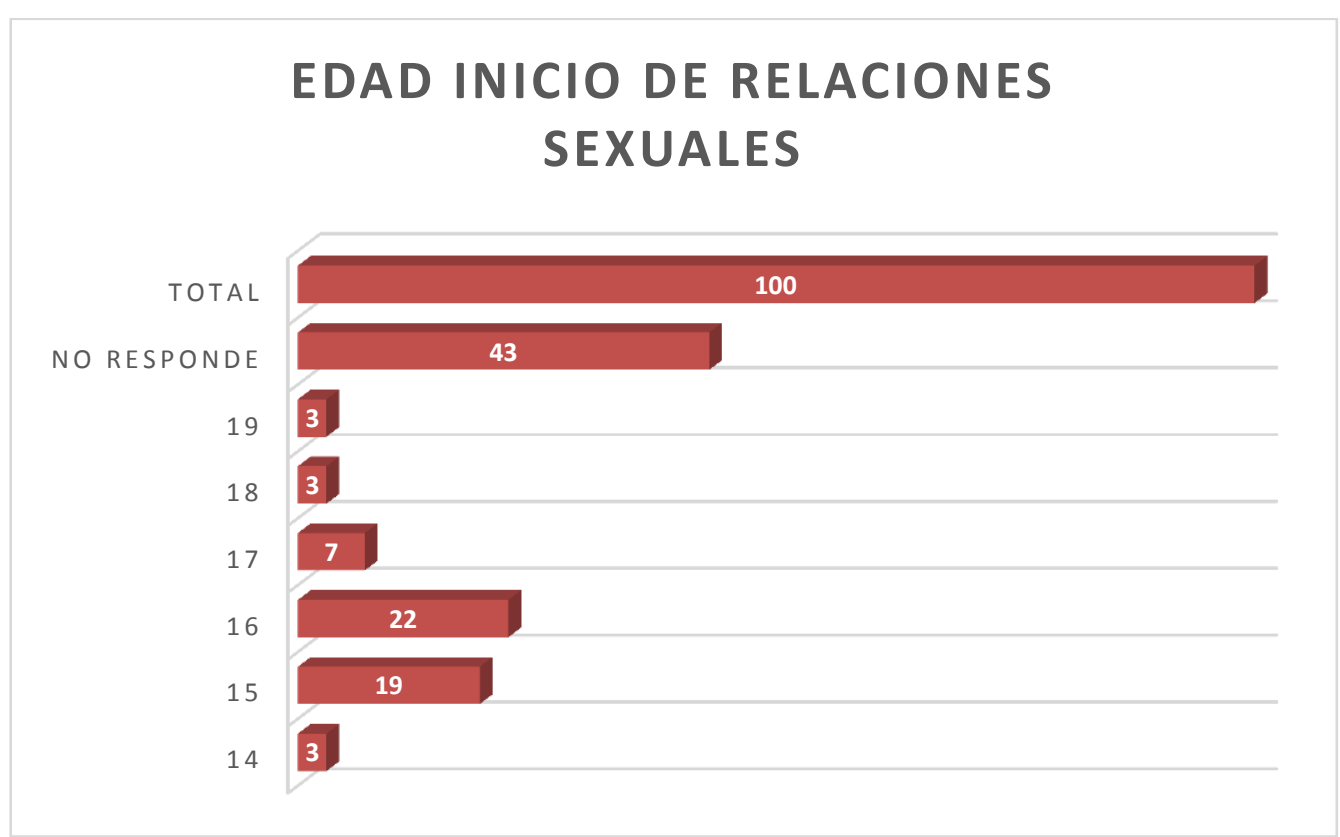

Gráfico 4. Edad de inicio de las relaciones sexuales 
De un $100 \%$ de los adolescentes encuestados al relacionar la edad con el inicio de las relaciones sexuales se obtuvo que la gran mayoría de la población no respondió a esta pregunta, de los que respondieron se obtuvo que un $3 \%$ inicio sus relaciones sexuales a los 14 años de edad, en igual porcentaje respondieron los adolescente de 19 y 18 años de edad, los que iniciaron relaciones sexuales a los 15 años representan el 19\% y los que iniciaron a los 16 años constituyen el 22\%, los adolescentes que iniciaron relaciones sexuales a los 17 años representaron el $7 \%$.

Al realizar la comparación de los gráficos anteriores con este gráfico 4 se puede observar que los adolescentes comienzan sus relaciones sexuales entre los 14 y los 16 años de edad y son los que poseen en mayor porcentaje los conocimientos incorrectos acerca de los métodos anticonceptivos lo que ocasiona un aumento en los casos de embarazos en adolescentes y la propagación de infecciones de transmisión sexual.

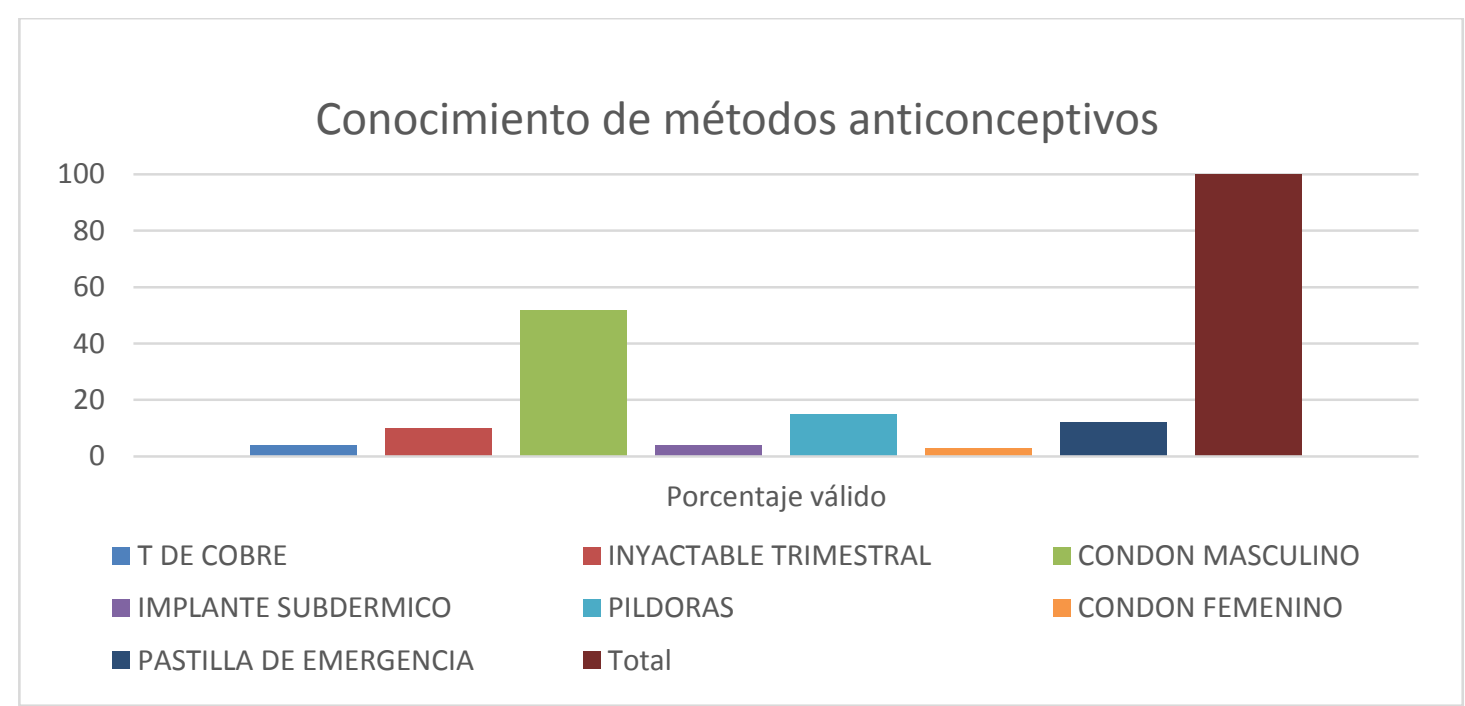

Gráfico 5. Conocimiento acerca de los métodos anticonceptivos

Al consultar acerca de los métodos anticonceptivos conocidos por los adolescentes en estudio se obtuvo que un $56 \%$ conoce acerca del condón como método anticonceptivos, un $15 \%$ conoce acerca de la pastilla de emergencia como forma de anticoncepción, un $12 \%$ conoce acerca de las píldoras, un $10 \%$ posee información acerca de la inyección trimestral como método anticonceptivo, en igual porcentaje $(4 \%)$ conoce acerca de la $\mathrm{T}$ de cobre y del implante subdermico y solo $3 \%$ posee información acerca del condón femenino. Es decir, la información más manejada por estos adolescentes es acerca del uso del condón masculino como método anticonceptivo. 


\section{Uso correcto del condon}

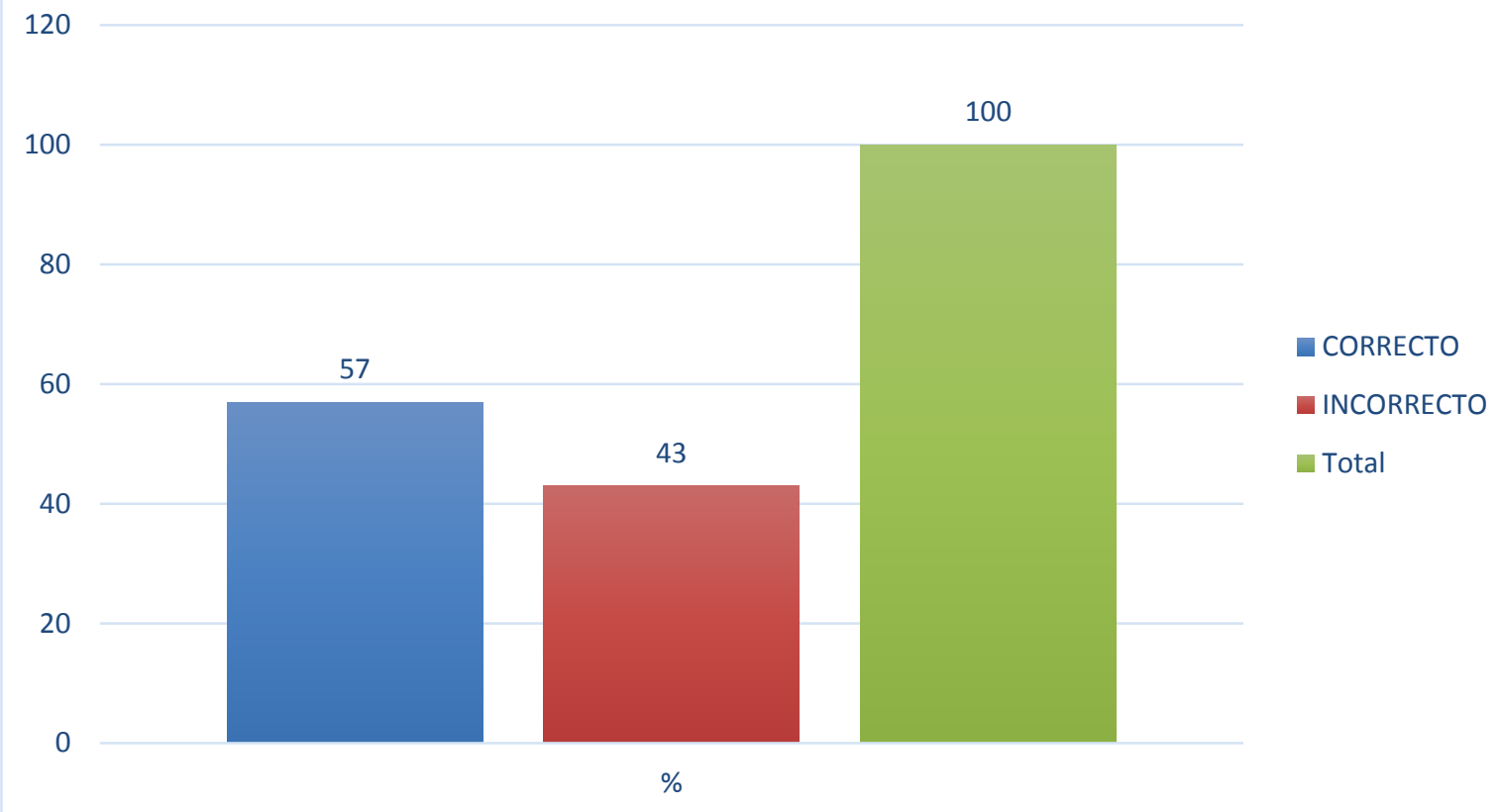

Gráfico 6. Uso correcto del condón masculino

Según el gráfico 5 el método anticonceptivo más conocido fue el condón masculino se indagó acerca de la información que poseen los adolescentes al respecto y se obtuvo que solo un $57 \%$ de estos adolescentes posee información correcta acerca de su uso y un $43 \%$ posee información incorrecta acerca del uso de este.

En este estudio, el análisis de los resultados determinó que el índice de conocimiento de los métodos anticonceptivos en esta población adolescente es bajo; si bien es necesario continuar mejorando la calidad de la información de los métodos anticonceptivos entre los adolescentes, también es necesario continuar fomentando el uso adecuado.

De los diferentes tipos de métodos anticonceptivos disponibles en los servicios de salud se pudo observar que los más usados son los condones masculinos y las pastillas de emergencia, métodos relativamente accesibles y fáciles de usar, pues ambos son gratuitos en las unidades de salud, sería importante pues realizar una investigación con respecto a las causas de no solicitar estos métodos.

En América Latina y el Caribe, 90\% o más de las adolescentes conocen al menos un método anticonceptivo, excepto Bolivia (74\%), Guatemala (68\%) y Paraguay (89\%), Sin embargo, los porcentajes de uso son bajos (18\%) (9).

En América Latina, las adolescentes han duplicado su aporte a la fecundidad total, pasando a representar un 14,3\%, (Comisión Económica para América Latina y el Caribe - CEPAL) (10).

La fecundidad en adolescentes bolivianos entre 15 y 19 años: era de 14\% en 1998, se incrementó a $19 \%$ el 2003 , siendo este porcentaje mayor en adolescentes con bajos niveles de instrucción; además la necesidad insatisfecha en anticoncepción que llega al 38\% según el de la ENDSA 2008 (11).

La Encuesta Nacional de Adolescencia y Juventud (ENAJ 2008), realizada en 17 ciudades capitales e intermedias, revela también datos muy importantes en 
relación a este tema. Aunque el conocimiento sobre métodos anticonceptivos entre adolescentes hombres y mujeres es relativamente alto ( 4 de cada 5 adolescentes y jóvenes conocen algún método anticonceptivo), su uso es relativamente bajo. El 37\% de mujeres entre 15-19 años usa un método anticonceptivo. Además, es importante resaltar que, aunque el 92\% de adolescentes entre 15-19 años conoce el condón, sólo el $29 \%$ lo utilizó durante su primera relación sexual (12).

Con los datos obtenidos en este estudio es importante realizar constantemente intervenciones educativas, para fomentar el uso continuo de métodos anticonceptivos, desde la primera relación sexual.

\section{CONCLUSIÓN}

os adolescentes de estratos sociales

$\mathrm{L}$ medios demostraron poseer información incorrecta acerca de los métodos anticonceptivos, aun cuando existe una variedad de MA disponibles actualmente el más conocido resultó ser el condón masculino, no obstante, la información que poseen de este es inadecuada, lo que puede ocasionar mal uso de este con las consecuencias que genera una inadecuada protección durante las relaciones sexuales. Es preocupante que con un promedio de inicio de la actividad sexual entre los 15 y 16 años de edad, no tengan los jóvenes la información acerca de los métodos anticonceptivos por el contrario la mayor información la poseen los adolescentes entre los 17 y 19 años de edad. Se pudiera señalar que a menor edad menor información posee la población estudiada.

La OMS ha señalado que, los adolescentes experimentan cambios físicos, hormonales, psicológicos y sociales que deben ser atendidos y a nivel global, una gran cantidad de adolescentes son sexualmente activos y la mayoría no utiliza ninguna protección para evitar el embarazo, enfermedades de transmisión sexual o el virus de inmunodeficiencia humana. Esta es una realidad preocupante en América Latina y el Caribe, de allí que resulta primordial reforzar los conocimientos de este grupo etario. La precitada consideración más los datos que arrojo la investigación dan cuenta de la necesidad de planificar y ejecutar programas educativos que tomen en cuenta las características de la población a la cual van a ser destinados, dirigidos a mejorar el dominio cognitivo acerca de los diferentes métodos anticonceptivos que existen y con ello favorecer una sexualidad responsable. La institución educativa y la familia juegan un rol de vital importancia para proteger la salud del adolescente promoviendo espacios de comunicación abierta y respetuosa, donde esta sienta que puede ser comprendido y no juzgado en su sexualidad.

- Conflicto de intereses. Ninguno declarado por la autora.

- Financiación. Ninguna declarada por la autora.

- Agradecimiento: Ninguno manifestado por la autora.

- Investigación realizada considerando los tratados bioéticos.

\section{REFERENCIAS BIBLIOGRÁFICAS}

1. Fernández B, Mariño E, Ávalos M, Baró V. Información sobre anticoncepción y métodos anticonceptivos en adolecentes del municipio "La Lisa". Rev Cubana Med Gen Integr [Internet]. 2013 Mar [citado 2018 Mar 05]; 29( 1 ): 3-7. Disponible en: http://scielo.sld.cu/scielo.php?script= sci_arttext\&pid=S0864-

21252013000100002\&lng=es

2. Vázquez F, Gómez B, Espinosa A, Camacho J, López J, Ulloa J. Nivel de conocimiento sobre métodos anticonceptivos en adolescentes. Research Gate 2017 nov 17; 27: 198812 
3. Organización Mundial de la Salud [Internet]. Salud de la madre, el recién nacido, del niño y del adolescente [consultado 23 May 2018]. Disponible en:

http://www.who.int/maternal_child_a dolescent/topics/adolescence/dev/es 4.

Montero V.

Anticoncepción en la adolescencia. Rev Med Clin. Condes. 2011[consultado 23 May 2018].; 22, (1):59 - 67. Disponible en: https://www.sciencedirect.com/scien ce/article/pii/S0716864011703935

5. Muñoz M. Anticoncepción en la adolescencia y casos especiales. Rev Esp Endocrinol Pediatr 2015[consultado 20 May 2018].; 5361.

Disponible en:https://www.endocrinologiapediat rica.org/revistas/P1-E12/P1-E12-

S510-A284.pdf

6. Sam-Soto $\mathrm{S}$, Osorio-Caballero $\mathrm{M}$, Rodríguez-Guerrero R. Comportamiento sexual $\mathrm{y}$ anticoncepción en la adolescencia. Acta pediátrica de México.2014. (Consultado 12 abril 2017); 35(6): 490-498.

Disponible en:http://www.scielo.org.mx/scielo.p hp? script=sci_arttext\&pid=S0186-

7. Soriano $\mathrm{H}$, et al. Criterios de Elegibilidad de Métodos Anticonceptivos: Nuevas Recomendaciones. Rev Clín Med Fam. 2010[citado 2018 Mar 06; 3, (3): 206216 Disponible en: http://scielo.isciii.es/scielo.php?script =sci_arttext\&pid=S1699. 695X2010000300009\&lng=es
8. Organización Mundial de la Salud. Criterios médicos de elegibilidad para el uso de anticonceptivos. 4⿳亠丷a Edición. Ginebra 2009. Disponible en: https://www.who.int/reproductivehe alth/publications/family_planning/Ex -Summ-MEC-5/es/

9. Maturana C, Álvarez J, Carbonel W, Goyeneche J. Sexualidad y métodos anticonceptivos en estudiantes de educación secundaria. Acta Médica Peruana (Internet). 2009. [citado 2018 Mar 06]; 26: 175-179.

Disponible en: http://www.scielo.org.pe/scielo.php? script=sci_arttext\&pid=S172859172009000300006\&lng=e

10. Valero P, Rausell D, Pacheco C, García J. Planificación Familiar Información General y Seguimiento de Anticonceptivos Orales. Med. esp. Disponible en: http://www.san.gva.es/documents/2 46911/251004/guiasap029planfam.p $\mathrm{df}$

11. Instituto Nacional de Estadística y Ministerio de Salud y Deportes, Encuesta Nacional de Demografía y Salud 2008, Bolivia, 2009.

12. Viceministerio de Igualdad de Oportunidades, Fondo de Población de las Naciones Unidas, Encuesta Nacional de la Adolescencia y Juventud 2008, Bolivia, 2009 